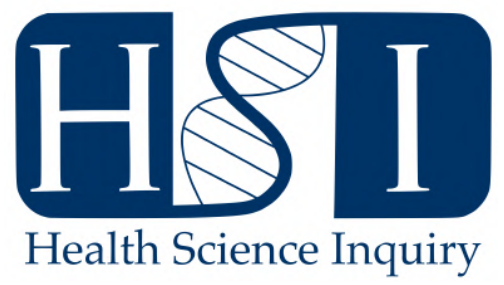

\title{
Altered State of Mind: Direct Cellular Reprogramming as Potential Treatment Strategy for Alzheimer's Disease
}

\author{
Anika Spasov ${ }^{1}$ and Dr. John Paul Oliveria ${ }^{2}$ \\ ${ }^{1}$ Department of Medicine, Division of Respirology, McMaster University \\ ${ }^{2}$ School of Medicine, Department of Pathology, Stanford University
}

\section{INTRODUCTION}

Reversing degenerative diseases and injuries has been a long sought out possibility in the field of regenerative medicine. While the notion that neurons could continuously regenerate throughout life was demonstrated just over twenty years ago, little substantial development has been made in the treatment of neurodegenerative diseases [1]. The question is asked, could neuroregeneration be induced in the suffering brain and be used to treat Alzheimer's disease (AD)? Nearly a decade before this discovery of neuroregeneration, the very first direct reprogramming of terminally differentiated cells into a cell type of different lineage was demonstrated via the conversion of fibroblasts into myoblasts. By inducing ectopic expression of MyoD, fibroblasts were successfully converted into myoblasts, without passing through a pluripotent stage [2]. As the understanding of both $\mathrm{AD}$ and cellular reprogramming has grown over time, the ability to promote regeneration or repair within the adult human brain has become a real possibility [3].

\section{Alzheimer's Treatment}

In particular, $\mathrm{AD}$ appears to be a promising target for direct cell reprogramming. AD is a chronic neurodegenerative disease characterized by neuroinflammation and the accumulation of disease-associated proteins in the brain, such as amyloid beta and neurofibrillary tau tangles, which ultimately leads to neuronal loss and synaptic degradation. The consequences of this degradation include cognitive dysfunction, motor impairment and memory loss, hence the quality of life of AD patients is greatly depleted [4]. Part of regulating the inflammatory response in $\mathrm{AD}$ involves anisomorphic astrogliosis: the increase and change in morphology of reactive astrocytes, leading to glial scar formation and microcircuit instability [4]. This pathogenic astrogliosis not only harms astrocytes, but also neighbouring neurons. Consequently, the repair and regeneration of neurons damaged by neural inflammation in $\mathrm{AD}$ have long been envisioned. The ability to produce neurons from human induced pluripotent stem cells (hiPSCs) has introduced the possibility of cellular transplants; however, the process is time and material intensive, while also raising ethical concerns regarding the use of stem cell technology [5]. Direct

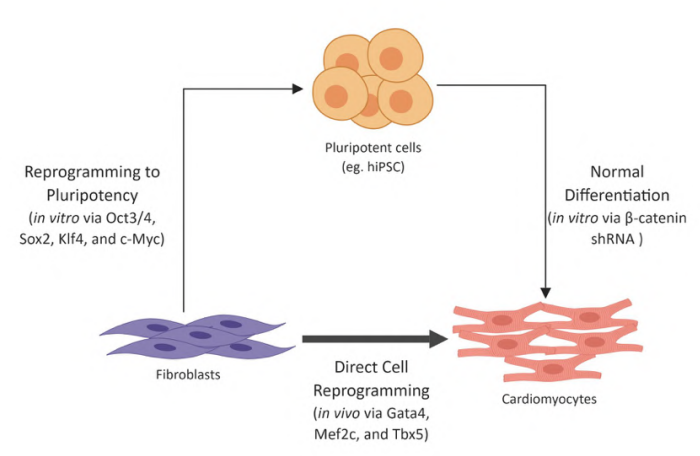

Figure 1: Terminally differentiated cells can be reprogrammed to a pluripotent stage, at which point the cells can go down a different cell lineage than previously. Direct cell reprogramming bypasses pluripotency and permits cells to directly convert from one cell type to another via ectopic expression of transcription factors or small molecule application. This method is more efficient and does not require in vitro culturing of pluripotent cells.

cell reprogramming bypasses the intermediate pluripotent stage and directly converts one cell type to another (Figure 1). Recent developments in pioneer transcription factors, such as FOX which binds directly to condensed chromatin, as well as small molecules capable of initiating direct reprogramming, demonstrate a more efficient means of replacing cells damaged by $\mathrm{AD}[3,6]$. By omitting the pluripotent step, the reprogramming process becomes quicker and more economically feasible. Additionally, the risk of teratoma development normally associated with hiPSCs is curtailed by circumventing pluripotency, while also averting the ethical controversy surrounding embryos and stem-cell therapy. Thusly, re-establishing diseased neurons, and possibly restoring function, has become an intriguing possibility in AD therapeutics through the development of direct cell reprogramming $[3,7]$. 


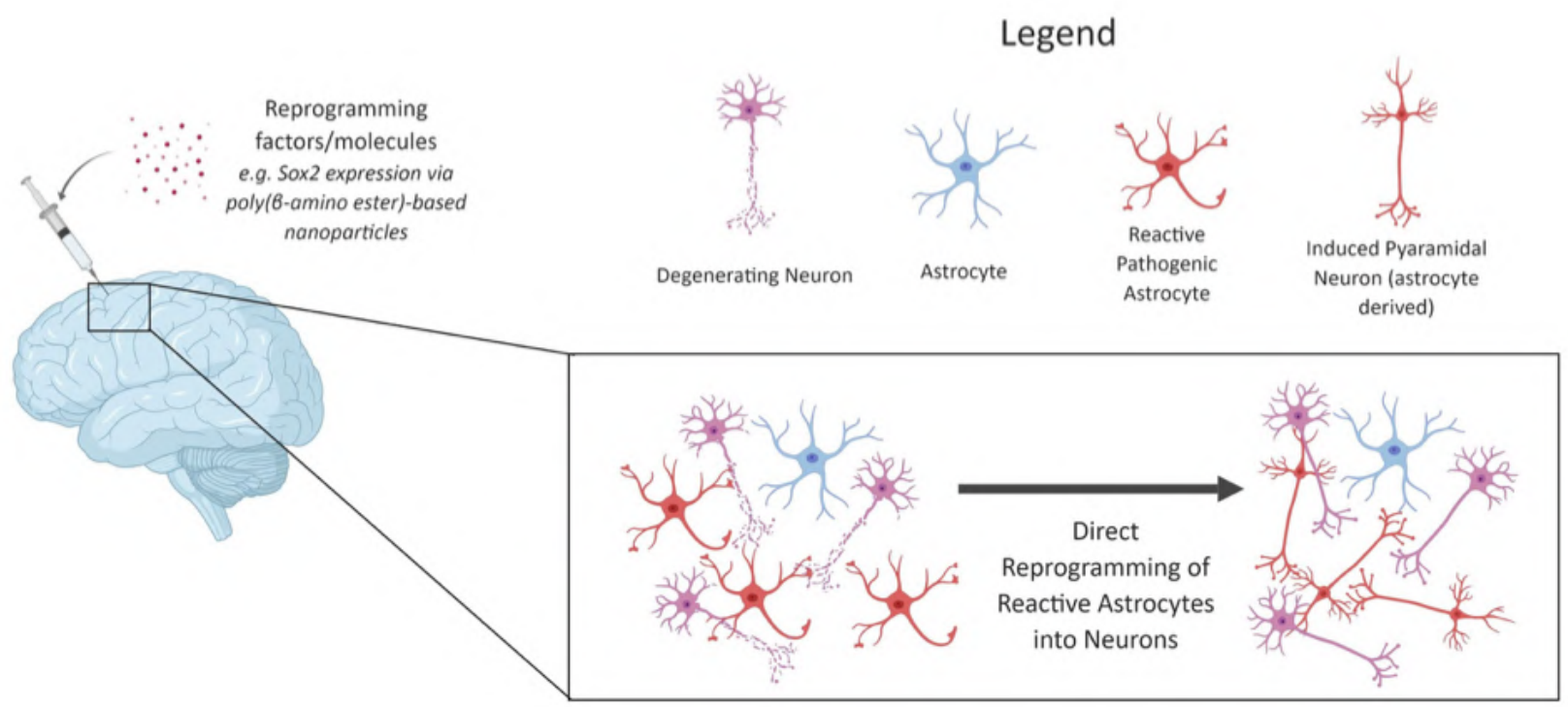

Figure 2: Reactive astrocytes undergo astrogliosis and are more easily converted via direct reprogramming, thus potentially pathogenic astrocytes could be transformed into functioning neurons. This may introduce a novel treatment of Alzheimer's by both preventing future damage from unwarranted astrogliosis and the regeneration of neuronal connections. Consequently, direct cell reprogramming of astrocytes may aid in tackling both the underlying pathology and regeneration of lost neurons in those afflicted with $\mathrm{AD}$.

\section{In vivo Neural Reprogramming}

A unique trait of direct cell reprogramming is the ability to perform the process in vivo [7]. Unlike hiPSCs, which must be removed and reprogrammed in vitro, direct cell reprogramming techniques can be applied to cells within their local tissue environment. It has been demonstrated to be possible in a multitude of organs: in the heart induced cardiomyocytes were formed, in the pancreas induced islet cells, and induced neurons have been observed in murine brains. The latter of this is most pertinent to $\mathrm{AD}$ and provides evidence for direct cellular reprogramming as a future therapy [7]. Sox2 alone is capable of converting reactive glial cells into neurons within the adult mouse cerebral cortex post-injury [8]. Even more substantial was the confirmation, via patch clamps, that these induced neurons received synaptic inputs from neighbouring cells, suggesting they may be cognitively functional and further elucidating direct reprogramming as a potential AD therapeutic [8]. Considering regions of the brain are difficult to access surgically and the inflammatory nature of $\mathrm{AD}$, it is also extremely relevant that in vivo direct reprogramming is much less invasive and virtually eliminates concerns of immunocompatibility commonly associated with donor hiPSCs [6].

Within glial cell reprogramming, astrocytes have been especially auspicious in regards to successful direct cell reprogramming into neurons. This, in addition to the large astrocyte population in the brain, suggest astrocytes would make particularly viable targets for reprogramming as a potential AD treatment. In a period of one to three weeks, a $90 \%$ astrocyte-to-neuron conversion efficiency was reached in mouse cerebral cortex [9]. This was achieved by com- bining Bcl-2 expression with antioxidative therapies, consequently reducing reactive oxidative species-induced apoptosis. Thusly, astrocytes are an extremely promising target for reprogramming technologies in the treatment of $\mathrm{AD}$, in comparison to other glial cells. Moreover, the generated neurons attained a pyramidal neuron morphology, thus suggesting they may be capable of advanced cognitive functions [9]. Additionally, within AD models, reactive astrocytes reprogram more effectively in vivo than unreactive astrocytes [10]. Clinically, this may be beneficial: by transforming reactive astrocytes into functioning neurons it could not only help restore neuron damage, but it can diminish the pathogenicity and inflammation resulting from astrogliosis (Figure 2). Converting adverse, reactive astrocytes into neurons prevents those cells from contributing to AD progression and thereby minimizes damage occurring in the brain [10]. Consequently, direct cell reprogramming is a two-pronged AD treatment, which not only regenerates new neurons, but also disposes of harmful reactive astrocytes.

\section{Challenges and Conclusions}

While direct cell reprogramming is a promising technology for the future of regenerative medicine, particularly in terms of neurodegenerative diseases, it still remains to be tested in the human brain and requires further research before being introduced as a clinical AD therapy. The ideal method of delivery still remains elusive and is a challenge that first must be met [7]. Viral vectors have been used most commonly in vivo; however, they risk transfecting neighbouring cells and causing ectopic expression in unintended cells. Recently, small molecules have been iden- 
tified which have the ability to directly induce neurons in vivo [3]. These face the challenge of remaining at targeted therapeutic concentrations over long periods of time. Howbeit, the utilization of nanoparticles in conjunction with these small molecules may mitigate this barrier by permitting longer exposure of treatment over time, within a single dose [7]. Moreover, future research should further analyze the functionality of these induced neurons and investigate if in vivo direct reprogramming can be validated within a human context [6]. All these challenges considered, direct cell reprogramming is a promising treatment for $\mathrm{AD}$ and has a place within the rapidly dynamic landscape of future health and therapies.

\section{REFERENCES}

[1] Eriksson PS, Perfilieva E, Björk-Eriksson T, Alborn AM, Nordborg C, Peterson DA, et al. Neurogenesis in the adult human hippocampus. Nature medicine. 1998;4(11):1313.

[2] Davis RL, Weintraub H, Lassar AB. Expression of a single transfected cDNA converts fibroblasts to myoblasts. Cell. 1987;51(6):987-1000.

[3] Xu J, Du Y, Deng H. Direct lineage reprogramming: strategies, mechanisms, and applications. Cell stem cell. 2015;16(2):119-134.

[4] Mohamet L, Jones VC, Dayanithi G, Verkhratsky A. Pathological human astroglia in Alzheimer's disease: opening new horizons with stem cell technology. Future Neurology. 2018;13(2):87-99.

[5] Fang Y, Gao T, Zhang B, Pu J. Recent advances: decoding alzheimer's disease with stem cells. Frontiers in aging neuroscience. 2018;10:77.

[6] Barker RA, Götz M, Parmar M. New approaches for brain repair - from rescue to reprogramming. Nature. 2018;557(7705):329.

[7] Srivastava D, DeWitt N. In vivo cellular reprogramming: the next generation. Cell. 2016;166(6):13861396.

[8] Heinrich C, Bergami M, Gascón S, Lepier A, Viganò F, Dimou L, et al. Sox2-mediated conversion of NG2 glia into induced neurons in the injured adult cerebral cortex. Stem cell reports. 2014;3(6):1000-1014.

[9] Gascón S, Murenu E, Masserdotti G, Ortega F, Russo GL, Petrik D, et al. Identification and successful negotiation of a metabolic checkpoint in direct neuronal reprogramming. Cell stem cell. 2016;18(3):396-409.

[10] An N, Xu H, Gao WQ, Yang H. Direct conversion of somatic cells into induced neurons. Molecular neurobiology. 2018;55(1):642-651.
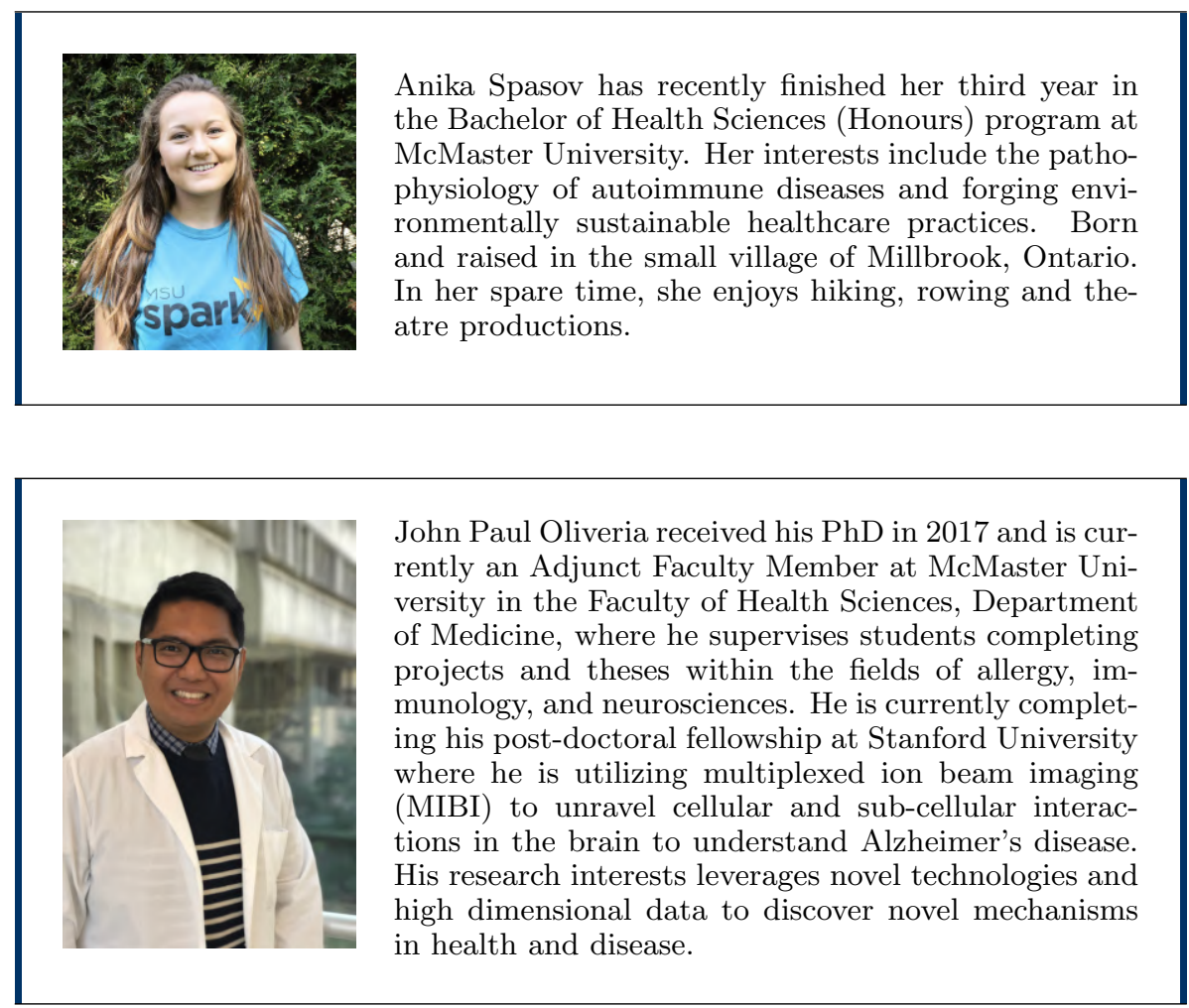